\title{
Immunotherapy and pulmonary toxicities: can concomitant immune-checkpoint inhibitors with radiotherapy increase the risk of radiation pneumonitis?
}

\begin{abstract}
To the Editor:
Delaunay et al. [1] presented the results of a retrospective study of 1826 cancer patients treated with immune-checkpoint inhibitors (ICIs). 3.5\% of the patients developed interstitial lung diseases (ILDs). The mechanisms of this toxicity remain largely unknown [2]. The cohort was mainly composed of lung cancer patients (75\%). This explains why a significant proportion of the patients (40\%) with ILD had received thoracic radiotherapy. However, patients with ILD features within the initial volumes of radiotherapy were excluded from the study to dissociate cases related to radiation pneumonitis. This study is important and brings to the light key data regarding the emerging field of clinical management of adverse ICI events.

It has been demonstrated that radiotherapy can prime the immune response [3] and thereby overcome the mechanisms responsible for resistance for immunotherapy; however, uncertainties remain regarding a potential synergy resulting in increased toxicities by combining the two treatments. Effects of concomitant ICIs with thoracic radiotherapy on pulmonary toxicities is not currently known. The KEYNOTE-001 trial studied the use of pembrolizumab for patients with advanced non-small cell lung cancer [4]. A secondary analysis of this trial compared the results according to whether the patient had received any prior radiotherapy. Pulmonary toxicities of any grade were observed more frequently in the patients who had received previous thoracic radiotherapy $(63 \%)$ versus those with no previous thoracic radiotherapy (40\%). No differences in grade $\geqslant 3$ pulmonary toxicity were noted. In the PACIFIC study of durvalumab versus placebo in stage III non-small cell lung cancer, rates of grade 3-4 pneumonitis were similar in both groups (3.4\% in the durvalumab arm and $2.6 \%$ in the placebo arm) [5]. However, these studies did not assess the toxicities of concomitant radiotherapy with ICIs; patients were treated sequentially with radiotherapy then ICIs. We report here two cases of patients treated with concomitant ICIs and stereotactic body radiotherapy (SBRT) who developed a radiation pneumonitis.
\end{abstract}

A 70-year-old patient with a metastatic melanoma was treated for a spleen metastasis with SBRT delivering $24 \mathrm{~Gy}$ (four fractions of $6 \mathrm{~Gy}$ ). Radiotherapy was administered during anti-PD-1 therapy, between the 21 st and the 22nd injections of pembrolizumab. 5 months later, high-resolution computed tomography (HRCT) showed peripheral consolidation in the left lower lobe within the fields of radiotherapy (figure 1). The findings were consistent with organising pneumonia and considered to be radiation pneumonitis. The patient was asymptomatic and has remained so since.

A second patient, a 59-year-old with a metastatic colon cancer (liver and lung metastasis), was treated with SBRT delivering $45 \mathrm{~Gy}$ in three fractions of $15 \mathrm{~Gy}$. SBRT was delivered concomitantly the day of the second injection of atezolizumab (anti-PD-L1). He developed a cough without dyspnoea 3 months later. HRCT showed patchy central areas of consolidation in right upper and lower lobes within the field of radiotherapy and beyond (figure 1). Bronchoalveolar lavage was performed, which revealed lymphocytes in the fluid without bacteria. The diagnosis of radiation pneumonitis was confirmed and steroid was prescribed at a dose of $1 \mathrm{mg} \cdot \mathrm{kg}^{-1}$. Notably, neither of these two patients had a history of smoking or chronic pulmonary disease. 

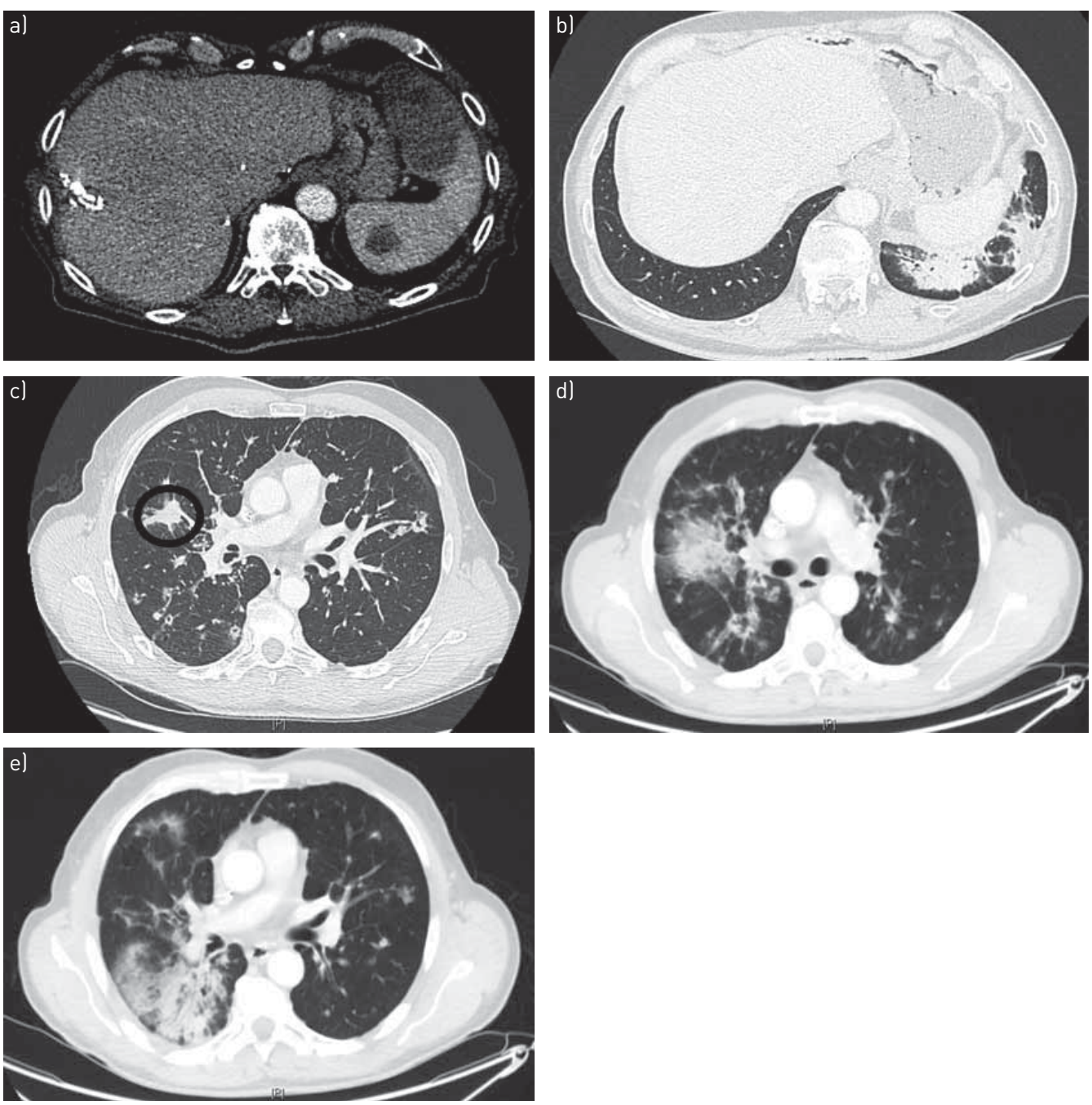

FIGURE 1 a) Case of a patient with a spleen metastasis of a melanoma who developed b) opacities of the left lower lobe 5 months after concomitant radiotherapy and anti-PD-1 therapy. Axial reconstruction of volumetric high-resolution computed tomography (HRCT) showed peripheral consolidation in the left lower lobe and ground-glass opacities. c) Case of a second patient with lung metastasis of a colon cancer: the initial HRCT showed lung lesion (black circle) that was treated by radiotherapy during immunotherapy (anti-PD-L1). Volumetric HRCT performed 3 months after irradiation showed unilateral peribronchial areas of consolidation mainly in the d) middle and upper lung zones and e) beyond.

Approximately $11 \%$ of patients develop radiation pneumonitis of grade $\geqslant 2$ within the few months after SBRT [6]. Cytokines and inflammatory molecules are known to be involved in the tissue developing radiation pneumonitis and lung fibrosis [7]. Transforming growth factor $\beta$ and Tumour necrosis factor $\alpha$ have been shown to play a central role in the genesis of this toxicity along with several interleukins (ILs): IL-4, IL-6 and IL-17A. While cumulative data underscore the pivotal role of cellular compartments such as macrophages and fibroblasts [8], so far, little is known regarding the impact of manipulation of T-cells. Radiotherapy is known to activate several key elements of the immune response: upregulation of expression major histocompatibility complex class I molecules, liberation of antigenic peptides, activation of dendritic cells and diminution of the regulatory T-cells [9]. In our institution, we have treated six patients with concomitant ICIs and SBRT; two cases of radiation pneumonitis occurred and are presented in this article. It is uncertain whether these two cases were just related to SBRT or if the combination with ICIs played any enhancing role. No clinical data are available about effects of concomitant ICIs and lung SBRT; prospective data from large series regarding the safety of this association are needed. The antitumour effect of concomitant ICIs and radiotherapy is still under investigation, and an important field of research into the induction of an effect on the irradiated and nonirradiated tumour lesions (the so-called abscopal effect). Although we cannot draw firm definitive, conclusions because of the small sample size of our series, we do believe that a particular attention should be paid to the diagnosis and 
management of these new toxicities associated with immunotherapy specifically in association with radiotherapy, and that forthcoming clinical trials should carefully record those events.

Guillaume Louvel $^{1}$, Rastislav Bahleda ${ }^{2}$, Samy Ammari ${ }^{3}$, Cécile Le Péchoux ${ }^{1}$, Antonin Levy ${ }^{1}$, Christophe Massard $^{2}$, Jérôme Le Pavec ${ }^{4}$, Stéphane Champiat ${ }^{2}$ and Eric Deutsch ${ }^{1,2,3,5}$

${ }^{1}$ Dept of Radiotherapy, Gustave Roussy Cancer Campus, Paris-Saclay University, Villejuif, France. ${ }^{2}$ Drug Development Dept, Gustave Roussy Cancer Campus, Paris-Saclay University, Villejuif, France. ${ }^{3}$ Dept of Radiology, Gustave Roussy Cancer Campus, Paris-Saclay University, Villejuif, France. ${ }^{4}$ Laboratoire de Chirurgie Expérimentale-UPRES (EA-2705), Centre chirurgical Marie Lannelongue, Le Plessis Robinson, France. ${ }^{5}$ Université Paris-Sud, université Paris-Saclay, faculté de médecine du Kremlin-Bicetre, Le Kremlin-Bicetre, France.

Correspondence: Guillaume Louvel, Dept of Radiation Oncology, Gustave Roussy, 114 Rue Edouard Vaillant, 94805 Villejuif Cedex, France. E-mail: guillaume.louvel@gustaveroussy.fr

Received: Aug 232017 | Accepted after revision: Sept 272017

Conflict of interest: Disclosures can be found alongside this article at erj.ersjournals.com

\section{References}

1 Delaunay M, Cadranel J, Lusque A, et al. Immune-checkpoint inhibitors associated with interstitial lung disease in cancer patients. Eur Respir J 2017; 50: 1700050.

2 Montani D, Seferian A, Parent F, et al. Immune checkpoint inhibitor-associated interstitial lung diseases: some progress but still many issues. Eur Respir J 2017; 50: 1701319.

3 Twyman-Saint Victor C, Rech AJ, Maity A, et al. Radiation and dual checkpoint blockade activate non-redundant immune mechanisms in cancer. Nature 2015; 520: 373-377.

4 Shaverdian N, Lisberg AE, Bornazyan K, et al. Previous radiotherapy and the clinical activity and toxicity of pembrolizumab in the treatment of non-small-cell lung cancer: a secondary analysis of the KEYNOTE-001 phase 1 trial. Lancet Oncol 2017; 18: 895-903.

5 Antonia SJ, Villegas A, Daniel D, et al. Durvalumab after chemoradiotherapy in stage III non-small-cell lung cancer. N Engl J Med 2017; 377: 1919-1929.

6 Zhao J, Yorke ED, Li L, et al. Simple factors associated with radiation-induced lung toxicity after stereotactic body radiation therapy of the thorax: a pooled analysis of 88 studies. Int J Radiat Oncol Biol Phys 2016; 95: 1357-1366.

7 Kainthola A, Haritwal T, Tiwari M, et al. Immunological aspect of radiation-induced pneumonitis, current treatment strategies, and future prospects. Front Immunol 2017; 8: 506.

8 Bickelhaupt S, Erbel C, Timke C, et al. Effects of CTGF blockade on attenuation and reversal of radiation-induced pulmonary fibrosis. J Natl Cancer Inst 2017; 109: https://doi.org/10.1093/jnci/djw339.

9 Demaria S, Golden EB, Formenti SC. Role of local radiation therapy in cancer immunotherapy. JAMA Oncol 2015; 1: $1325-1332$. 